\title{
Dealing with constraint issues in agile discrete manufacturing companies
}

\author{
Hans Bonde and Hans Henrik Hvolby \\ Department of production, Aalborg University, Denmark, $\{$ hb, hhh $\} @ i p r o d . a u c . d k$
}

\begin{abstract}
Most manufacturing small and medium sized companies (SMEs) deal with constraint issues on a daily basis in terms of missing materials or lack of production capacity. Only a few of these companies have implemented Advanced Planning and Scheduling (APS) systems, which enables constraint based planning, as existing APS systems are aimed at the high-end market (e.g. Manugistics and 12) or part of large ERP suites (e.g. Oracle, SAP or BaaN). This means that the infeasible plans are sent to the shop floor, or that the planner has dealt with the constraint issues manually. Both situations are unacceptable in an agile manufacturing company.

This paper deals with planning in agile discrete manufacturing SMEs, with focus on the need for a well-suited planning system. It is argued that the quality of a plan depends on the throughput and the compliance with the overall business model. This leads to the argument that the role of the planning tool ideally is to calculate, while the planner makes all decisions on solving the constraint issues. The interaction between the planner and the planning tool is therefore of high importance, which again leads to new requirements for modern planning tools - and for the planner. Therefore the planning tool must provide the planner of information regarding the status in the production, which constraint issues needs to be solved etc.

The paper falls into four sections. First a comparison of lean and agile manufacturing environments is made to explain the different objectives of the planning. Secondly, a short section explains the current status of SMEs, outlining, how planners cope with constraints on a daily basis. Next is a section, which outlines some key points on, how the agile planning system should work. Lastly, the requirements for an agile planning/scheduling tool are identified.
\end{abstract}

\section{Keywords}

Planning and scheduling, APS, agile manufacturing, SMEs, planning tools.

\section{LEAN AND AGILE PLANNING}

In an effort to understand the requirements for the agile planning system, it is necessary, first to review these two different manufacturing paradigms, to understand the objectives of agile planning. 


\section{Lean manufacturing}

The main idea of lean manufacturing, which was described by Womack et al (1991), emerged in the Toyota factories in Japan in the 50's and 60's. The key is to manage the flow to eliminate waste in all parts of the production. By eliminating waste, fewer resources, i.e. materials, parts, capacity and employees are required to manufacture, which cuts costs significantly.

Lean is a paradigm, which includes the entire organization, and in the case of the automobile industry - the entire supply chain (Womack et al 1991). The most affected areas are product development and manufacturing. The keyword is wasteelimination. As lean manufacturing is cantered on controlling the flow, the flow needs to be balanced through the manufacturing unit - and the supplies to each link of the manufacturing process should be planned accordingly.

Compared to mass production, lean uses product families to increase the number of variants - and thereby the flexibility, without large loss of speed. Lean emerged together with the concept of JIT planning, i.e. a product is ordered when it is needed, and Kaizen - the concept of continuously making incremental improvements throughout the organization.

Combined these concepts calls for a planning system that supports the flow and keeps it moving. The objectives of the planning system are efficiency and speed. This is often obtained by producing in the largest possible batches, thereby minimizing setup times, while minimizing WIP and inventories. It is crucial that planning includes the entire flow of goods, as disturbance in one area of the flow affects the entire flow. Upstream, items are produced earlier than needed, and downstream, machines may become idle. It is a system, which is very sensible to disturbances in form of lack of materials, breakdowns etc. A problem in the flow affects the entire manufacturing unit.

\section{Agile manufacturing}

Goldman et al (1995) defines an agile company as a company, which is "capable of operating profitably in a competitive environment of continually, and unpredictably changing customer opportunities". This means that the keywords for an agile manufacturing company is customer focus and flexibility - i.e. the ability to respond to the changes in the environment in form of: fluctuating demand, increased demand for customized products, shorter delivery lead times, uncertainty of supply and increasing competition. This will, according to Hormozi (2001), mean that the concepts of agility will replace mass-production as a production paradigm.

Mønsted et al (2000) argue that the remedies for obtaining agility are flexible batch-sizes, reduced inventories, and more frequent deliveries. Hormozi (2001) points to short lead times, and the integration of design/engineering with manufacturing and marketing as ways of obtaining agility. Abdel-Malek et al (2000) list five types of flexibility that according to surveys are the most important: Machine flexibility, routing flexibility, process flexibility, product flexibility and volume flexibility.

For these reasons, the road to agile manufacturing includes technological and organizational changes as the foundation of agility, but the planning system is also 
affected by these changes. As the agile company is customer focused, the planning system must strive to meet customer/market demands rather than shop-floor demands, i.e. serve customer requirements instead of maximizing other key performance indicators (inventory turns, productivity, etc.). To make the planning system more customer-focused, Planning and re-planning must be done more frequently, which means that the speed of the planning process becomes a key factor. Moreover, the goal of planning shifts from internal efficiency to external service.

In order for the planning system to meet these requirements, it needs to support the goals of faster more customer focused planning, while respecting internal constraints in form of materials and capacity. A keyword for planning tools, aimed at SMEs is simplicity, e.g. in use, architecture and also planning methods. The planner also faces new challenges in an agile manufacturing company. Goldman (1995) stresses the importance of skilled employees, who are able to make decisions, based on the right information. This means that the planner must possess certain competencies in order to do the job. Secondly, the planner should be allowed to make choices, and there should be no organizational or technical barriers prohibiting this. Thirdly, the planner should be provided with the needed information to make the right decisions.

\section{Comparison of lean and agile planning}

Obviously, A shallow comparison between the two manufacturing concepts shows, that several areas do overlap, but a deeper look reveals that they are fundamentally different. Compared to the traditional mass production paradigm, they both claim to be customer-focused, increase flexibility, use multi-skilled resources with multiple functions, favour automation of manufacturing processes. They do however take different approaches to reaching these goals, and that also affects the way the planning system works.

Table 1 - Differences between agile and lean manufacturing and planning.

\begin{tabular}{|l|l|l|}
\cline { 2 - 3 } \multicolumn{1}{c|}{$\begin{array}{l}\text { Manufacturing } \\
\text { paradigm }\end{array}$} & $\begin{array}{l}\text { Flow oriented paradigm, } \\
\text { focused on eliminating waste in } \\
\text { each link of the manufacturing } \\
\text { process, while maintaining a } \\
\text { balanced flow. WIP, buffers } \\
\text { and inventories are considered } \\
\text { waste, and are sought } \\
\text { minimized. }\end{array}$ & $\begin{array}{l}\text { MTO oriented paradigm, focused } \\
\text { on flexibility, which is to produce } \\
\text { any number of any item at a } \\
\text { competitive price, quality and } \\
\text { delivery lead-time. Flexibility is } \\
\text { generated through machine, } \\
\text { routing, process, product and } \\
\text { volume flexibility. Buffers are } \\
\text { recognized as a means of } \\
\text { obtaining flexibility. }\end{array}$ \\
\hline Planning & $\begin{array}{l}\text { Highly integrated with JIT } \\
\text { planning, which uses a pull- } \\
\text { strategy in the entire process, } \\
\text { and thus, no raw material, part, } \\
\text { sub-assembly or finished } \\
\text { product should be produced }\end{array}$ & $\begin{array}{l}\text { The agile planning mixes push } \\
\text { and pull strategies to obtain } \\
\text { flexibility. The constraints may } \\
\text { shift often, depending on the } \\
\text { demand pattern, which means that } \\
\text { there is a need to identify them in }\end{array}$ \\
\hline
\end{tabular}




\begin{tabular}{|c|c|c|}
\hline & $\begin{array}{l}\text { before it should be used. The } \\
\text { causes the production process } \\
\text { to be highly sensitive to } \\
\text { disturbances. The constraints } \\
\text { largely remain the same over } \\
\text { time, and throughput can be } \\
\text { optimized on these }\end{array}$ & the planning process. \\
\hline $\begin{array}{l}\text { The planning } \\
\text { process }\end{array}$ & $\begin{array}{l}\text { Planning is often decentralized, } \\
\text { e.g. through a kanban } \\
\text { replenishment strategy. } \\
\text { Changes in one area affect the } \\
\text { entire upstream flow. Thus, the } \\
\text { planning is critical, and a time } \\
\text { fence is often used to remove } \\
\text { noise in the flow. }\end{array}$ & $\begin{array}{l}\text { Planning is more or less a } \\
\text { continuous process, focused on } \\
\text { serving the customer needs, and } \\
\text { thus the ability to change the plan } \\
\text { fast is a key parameter in agility. } \\
\text { There is a need to combine } \\
\text { central and local planning (van } \\
\text { Assen et al, 2000). Ideally, no } \\
\text { time fence is needed. }\end{array}$ \\
\hline
\end{tabular}

\section{THE CURRENT STATE IN SMES}

While companies may have made significant organizational and technical changes towards agility, they often lack proper planning tools that can enable agile planning. Older planning tools aimed at SMEs are relatively slow, and planning calculations are therefore often done in large batch jobs, most often performed at night. The result is that the plans are only updated once a day, which is not satisfactory in an agile company.

In most companies, a large part of the planners' day is spent dealing with capacity and material constraints. Moreover, most ERP systems aiming at small and medium sized enterprises (SMEs) use the MRP or MRPII planning technique under the premise of unlimited capacity. This premise results in several orders being planned on the same resource at the same time. This obviously creates infeasible plans, which are either changed manually or sent to the shop floor "as-is". Either way, the constraint issue is dealt with in the most typical ways of solving constraint issues are listed.

Table 2 - Common plan changes used by the planner to solve constraint issues

\begin{tabular}{l|ll}
\hline Solving capacity constraint issues & \multicolumn{1}{c}{ Solving material constraint issues } \\
\hline $\begin{array}{l}\text { Move to other planning period } \\
\text { (backwards/forwards) }\end{array}$ & Reduce order sizes \\
Minimize setup time & Use alternative materials \\
Overlap processes & Break up finished goods and use \\
Increase capacity (overtime) & materials \\
Cancel orders & Move to other planning period \\
Move to other capacity (internal or & (forwards) \\
external) & Cancel orders \\
Reduce order sizes & Change other products \\
& Request earlier deliveries (external or \\
\end{tabular}


When the plan is changed, orders are prioritized in order to maximize the company satisfaction level while obeying the constraints. The rules of prioritizing are closely connected with the overall business model, in which demand type (customer order, stock order), customer relationships, corporate culture, internal and external relations and other aspects play an important part. Thus, plan manipulation is a study of both quantitative and qualitative dimensions. There is however rarely an opportunity to identify the accurate consequences of a given manipulation, as it requires substantial information.

\section{WHERE TO GO}

It is obvious that SMEs need better planning tools, which not only supports $\mathrm{CBP}$, but also allows for increased interaction with the planner. However, in order to detail the requirements for the system, it is necessary to look deeper into CBP, knowledge requirements and shortcomings of current systems.

\section{Constraint based planning}

Constraint theory and the TOC philosophy - founded by Eli Goldratt (Goldratt \& Cox 1984) - is a philosophy, which claims that every system has one or more constraints that determine the throughput of the system. It is considered an overall theory for running an organization (Rahman 1998), but in terms of production planning, constraints are generally limited to be either materials/parts or resources (machines, tools, and employees). In order to perform CBP calculations, other factors are also considered to be constraints, e.g. due date, process time BOM and routing.

TOC consists of a five-step model which goal is to utilize the constraints as much as possible. The first step is to identify the constraint. The second step is to determine how to exploit the constraint, i.e. maximize the throughput. The third step is to subordinate "everything else" (Schragenheim \& Dettmer 2001), i.e. make sure that the rest of the system is focused on supporting the constraint. Fourth step is to elevate the constraint, i.e. increase the throughput capacity on the constraint, either generally or for a short period, if exploitation of the constraint does not solve the problems. Fifth step is to start over again.

To perform CBP, the constraints are divided into hard constraints and soft constraints. The hard constraints cannot be exceeded (elevated) while the soft constraints can (Steger-Jensen \& Hvolby 2001). The purpose of the planning is to respect the hard constraints, while the soft constraints can be exceeded if there is a need to do so. Most often, material availability is regarded a hard constraint, while due date, is a soft constraint. The capacity of the machines or employees is often considered a hard constraint, but can in some cases be exceeded, e.g. by working overtime or adding an extra shift. 


\section{Different ways of doing $\mathrm{CBP}$}

Finite loading means that the load of any resource must not exceed the capacity limit, i.e. orders may not be scheduled on the same resource at the same time. General MRP planning principles can be used, but if orders due to capacity constraints can not be placed in the desired time-frame or earlier, forward planning will be used. The benefit of this type of CBP is that it creates more feasible plans than ordinary MRP, in that capacity constraints are respected.

Planning on one constraint is a more complex matter. First, all constraints are identified and divided into hard and soft constraints. The machine with the highest load is the critically constrained resource (CCR). This machine is carefully planned for - in order to maximize the throughput. All other machines are subordinated to the CCR, i.e. their schedules depend on the schedules of the CCR - making sure that their capacity is not exceeded. This type of planning is simplified if all other resources are non critical capacity resources (non-CCRs), i.e. resources with loads below capacity or resources with process times lower than the CCR - which is often the case in e.g. a production line layout.

Planning on several constraints is planning on several machines, in order to maximize the throughput on the manufacturing unit as a whole. It requires advanced planning tools to identify and calculate for several constraints. These tools rely on calculation algorithms, which, as in the case of planning on one constraint, identifies the CCR decide how to exploit and subordinate.

Hsu and Chung (1998) present one such algorithm; a dominance rule-based algorithm, classifying non-critically constrained resources (non-CCR's) into three levels in order to solve a constraint problem - consisting of multiple products and multiple resources. As preparation the constraint issue is simplified by reducing the number of constraints through elimination of first and second level non-CCRs. After this, the constraint issue is solved in two steps.

w First, the constraint is identified, as the resource with the highest overload is identified. If no overload is present, there are no constraints, and the problem is solved.

w Second, the mix problem for the capacity constraint resource is solved, reducing the quantum of products, which has the lowest payoff/time on the constraint.

These steps are repeated until there are no resources with an overload. This algorithm is based on three assumptions:

- An economically optimized solution is the best solution.

- This assumption is fundamentally wrong. The result of an optimized plan is not necessarily in compliance with the overall business plan - and will therefore not maximize the company satisfactory level.

- Reducing the number of items produced is the way to solve constraint issues.

- None of the other plan changes used to solve constraint issues presented in

- are considered in the algorithm.

- All data needed to solve the constraint problem is available and valid.

- In order for this assumption to be correct, the company should have updated, valid data, which is very rare in SMEs. 
However, using the TOC algorithm leads to a product mix, optimized merely on economic aspects. It does not take the overall business-model into consideration, or tries other plan changes.

\section{REQUIREMENTS FOR AN AGILE PLANNING TOOL}

To sum up, a new planning tool, aimed at agile manufacturing SMEs, needs to support the planner. The basic idea is to have the tool perform the same thinking process, as a planner would, i.e. calculate on the plan changes, suggested in

. Moreover, the tool needs to...

- Be fast - and calculate plans many times a day.

- Identify constraints on the shop floor.

- Create feasible plans that can be followed on the shop floor.

- Monitor the progress on the shop floor - and take it into consideration when planning

- Provide information to the planner - not make decisions on solving constraint issues.

The needed functionality is presented by means of examples, which illustrate, which information, the tool should provide for the planner.

Example 1: provide information to the planner A regenerative or net change plan has been calculated, and the system has found that machine $\mathrm{A}$ is overloaded = constraint. The system should then evaluate how the problem can be solved, and provide the following information to the planner.

Example 1:

$<$ Machine $A>$ is overloaded five hours on $<$ date $>$

- Move to earlier date

- Orders cannot be moved to earlier date

- Move to <later date>, customer orders or stock replenishment orders will be affected.

- Moving prod.order 10000 will delay cust.order C100 to <customer> and $\mathrm{C} 101$ to < customer>

- Moving prod.order 10001 will delay cust.order C104 to <customer>

- Moving prod.order 10002 will delay stock refill, demand can be met by safety stock.

- Minimizing setup time will increase capacity

0 Changing prod.order 10000 and prod.order 10002 will reduce setup time by 0.5 hours

- Overlapping processes will not increase capacity

- Increase capacity by 5 hours

0 Increase two hours on <date> and three hours on <date>.

- Move production orders

0 Move prod.order 10000 - prod.time $=2$ hours - to $<$ machine $B>$

- Reducing order sizes will affect some customer orders

- Reduce prod.order 10000 will effect cust order $\mathrm{Cl} 100$ or $\mathrm{Cl} 101$

- Reduce prod.order 10001 with $\langle x\rangle$ items without affecting customer orders

- Reduce prod.order 10002 with $<y>$ items without affecting customer orders 
This will allow the planner to make decisions on how to solve the constraint issue. In this example, the solution might be to move production order 10002 to a later date, and the capacity increased to complete the other production orders.

To provide this information, the tool makes a series of calculations. In this example, the tool does not mix the possible plan changes, e.g. move production order 10002 to a later date and increase capacity to produce the remaining orders in one calculation. Such functionality requires rule-based calculation, which will be addressed later in this paper.

The example deals with planning on one constraint and it does not consider effects on other constraints (materials, machines, tools or employees) if a certain solution is chosen. If capacity is increased on machine $\mathrm{A}$, it is likely that another constraint will rise to be the CCR. This implies that the TOC algorithms presented by Hsu \& Chung (1998) and further developed by Onwubolu \& Mutingi (2001) are extremely important for constraint management, as they help calculate effects of a given manipulation. However, it is important to note that the solution space is not limited to adjusting the number of items planned, but should consider all possible changes listed in.

This improved action messaging or exception messaging functionality is key to providing the planner with state specific knowledge. It is however not sufficient, as it only provides information about the supply/demand pattern (with tracking information) and load on the constraints. This messaging does however not provide information on the status in the production unit.

Example 2: A machine breaks down. Machine A breaks down, and the system should automatically warn the planner by providing the following information.

Example 2

$<$ Machine A> is down. Operators guess for recovery is 4 hours

- The following orders are scheduled today

- Order 10000 for cust.order $\mathrm{C} 100$ to <customer> and C101 to <customer>

o Order 10001 for inventory.

o. Order 10002 for inventory.

- Some orders can be moved to other resources

- Orders 10000 and 10001 can not be moved

o Order 10002 can be moved to <Machine B>

- Some orders can be postponed

o. Order 10000 must be started no later than tomorrow at 12:00 to meet due date

- Order 10001 can be postponed 2 days

- Order 10002 is already late

To generate such information, the tool needs to have Supply Chain Event Management (SCEM) functionality. SCEM focuses on exceptions to the general workflow, that is, when something goes awry. It consists of five activities, defined by AMR Research (Bittner, 2000) as: 
a Mitor: Provide ongoing information about supply chain objects and events, including the current status of inventories, orders, shipments, production and supply.

- Notify: Support real-time exception management through alert messaging, which proactively warns a decision-maker if an action must be taken.

- Simulate: Support of decision making by assessing consequences of actions through simulation runs - often based on optimization methods.

- Control: The ability to proactively change a previous decision.

- Measure: Provide measurement on performance indicators (also KPIs) and metrics.

Thus, the operator needs to have easy access to register the information in the Manufacturing Execution System (MES) part of the planning tool - and that the planning tool needs to react to this information by notifying the planner, and e.g. create a new plan. Same sort of information should be provided for all exceptions in the production unit, i.e. lack of materials, orders that are not started/finished on time etc.

The goal of this feedback is to enable the planning system (both tool and planner) to react when the plan is not followed. The plan should always depict the actual activities of the manufacturing unit. A production order planned to start or finish "in the past" should therefore not be allowed.

\section{THE BALANCE BETWEEN RULE BASED PLANNING AND PLANNER DECISION MAKING}

As stated earlier in this paper, solving constraint issues, with the use of the plan changes presented becomes a complex matter, when they are mixed. This is however not the only problem encountered. When the number of constraints increases, so does the complexity of the problem, and with the requirement for more frequent planning runs, the time spent on planning will increase tremendously.

For the planning tool to solve the issue, presented in example 1, it must possess knowledge of how; the company wants to solve the problem. This means, that the possible plan changes presented in, needs to be prioritized.

This does however not alone solve the problem. In example 1 , an inventory replenishment order is postponed and customer orders are processed. This means that the solution is not only a matter of choosing the right plan change, but also a look at the order tracking to see, what demand it is to cover. If the production order is linked to a customer order, the planner will probably want to prioritize it higher than orders created to refill inventory. Lastly, the status on the shop floor is of concern. If the load on the plant is generally high, an increase of the capacity on machine A will not solve the problem. This will cause another machine to be the CCR, and thus create new problems.

The solution is therefore constrained by the possible plan changes, the order type/tracking information and the status on the shop floor. To allow the planning tool to support the planner with these choices - and thereby narrow the solution space, it must be possible to set up this information. 
Increasing the number of possible solutions to the constraint problem also increases the time the planner has to spend on evaluating the consequences and choosing the right solution, which makes it hard to create the plans fast. A planning tool that requires input from the planner every time a choice is made is therefore not suitable - as the planner becomes the new constraint. This is avoided with the use of rule-based systems, which evaluates the possible plan changes of and selects the best solution. The choice of the "best fit" solution is based on the prioritizing of orders; plan changes and the current status on the shop floor. This automatically reduces the number of solutions, decreasing the planner's time for evaluation and decision. It should also allow for combination of the plan changes as presented in example 1 . This requires, that the tool looks at the orders individually, follows tracking information to see, what the orders are for (customer vs. inventory replenishment orders).

There is obviously a conflict between the call for rule-based systems that makes choices for the planner and the statement that the planner makes all decisions. However in a manufacturing environment with many constraint issues, manual handling of each issue becomes too complex. With few constraints, the planner can cope with the issues. If the constraints are fixed, i.e. they always appear on the same resource, planning also becomes simpler. Recalling that a keyword for the tools aimed at SMEs is simplicity, it is a goal to use the simplest possible form of planning, as this will enable the planner to manage the planning process better.

Table 3 - The choice of planning tool and planning method depends on the number and placement of the constraints.

\begin{tabular}{|c|c|c|}
\hline & Few constraint issues & Many constraint issues \\
\hline Fixed & $\begin{array}{l}\text { E.g. a production line } \\
\text { environment, which is best, } \\
\text { supported by finite loading. The } \\
\text { bottleneck machine will set the } \\
\text { pace of the system - and the } \\
\text { planning tool need only solve the } \\
\text { constraint issue on that particular } \\
\text { machine. }\end{array}$ & $\begin{array}{l}\text { Typically a manufacturing environment } \\
\text { with large investments in several } \\
\text { expensive manufacturing equipment } \\
\text { This situation calls for rule based } \\
\text { planning, that automates the planners } \\
\text { choices. }\end{array}$ \\
\hline Moving & $\begin{array}{l}\text { A typical manufacturing } \\
\text { environment in many SMEs } \\
\text { producing large orders. The } \\
\text { bottleneck follows the order. } \\
\text { Manual handling of the } \\
\text { constraints is possible due to few } \\
\text { constraint issues. The rule based } \\
\text { narrowing of the solution space is } \\
\text { needed to support the planner. }\end{array}$ & $\begin{array}{l}\text { A typical situation when the company } \\
\text { falls behind on customer orders. The } \\
\text { best way to solve this situation is to use } \\
\text { optimization, which is also a rule based } \\
\text { planning method. } \\
\text { Simulation is another planning } \\
\text { methodology, which is very effective } \\
\text { for this environment. }\end{array}$ \\
\hline
\end{tabular}




\section{CONCLUSIONS}

A well suited planning tool is a key element in obtaining agility. The agile manufacturing company relies heavily on the planning tool when planning to meet customer demands. Currently, the planning tools do not support the planning activities satisfactorily, which causes the planner to manually change the output of the planning system to solve constraint issues. This is necessary as not many companies qualify for class A planning and that the output of the system does not always comply with the overall business model.

As these conditions are not likely to change in the future, the planning tool is to provide the planner with the information that enables him/her to change the plan, i.e. make it feasible. This means that optimization algorithms and calculations are necessary, but not adequate. Improved action messaging is the key to providing the planner with the information needed to create the best possible plans.

\section{REFERENCES}

[1] Abdel-Malek, Layek; Das, Sanchoy $\mathbb{K}$; Wolf, Carl, Design and implementation of flexible manufacturing solutions in agile enterprises, International Journal of Agile Management Systems; Volume 2 No. 3; 2000

[2] Bittner, M. (2000) E-Business Requires Supply Chain Event Management, AMR Research, Nov. 2000, www.amrresearch.com/Research/Reports/Pdf/001101Report13167.pdf, membership required

[3] Goldman, S.L., Nagel, R.N., Preiss, K. (1995) Agile competitors and virtual organizations: strategies for enriching the customer, Van Nostrand Reinhold, NY, ISBN 0-442-01903-3

[4] Goldratt, A., Cox, J., (1984), The goal, HighBridge Company, ISBN: 1565114248

[5] Hormozi, A.M. (2001) Agile manufacturing: the next logical step, Benchmarking: an International Journal, vol. 8, No. 2, pp. 132 - 143.

[6] Hsu, T., Chung, S. (1998), The TOC-based algorithm for solving product mix problems, Production planning \& Control, Vol. 9 No 1, pp. 36 - 46.

[7] Mønsted, L., Rasmussen, J., Skov, T., (2000), E-logistik, Børsens Forlag, ISBN 877553-836-9

[8] Rahman, S. (1998), Theory of constraints - a review of the philosophy and its applications, International Journal of Operations \& Productions management, vol. 18 No. 4 pp. $336-355$

[9] Onwobulu, G.C., Mutingi, M. (2001), A genetic algorithm approach to the theory of constraints product mix problem, Production planning and control, 2001, Vol 12, no. 1, pp $21-27$.

[10] Schragenheim, E, Dettmer, H.W., (2001) Manufacturing at Warp Speed: Optimizing Supply Chain Financial Performance, The St. Lucie Press/APICS series on constraint management.

[11] Steger-Jensen, K., Hvolby, H.H., (2001), Analysis of the planning and scheduling functionality in APS systems, Proceedings of SPIE conference 2001.

[12] van Assen, M.F., Hans, E.W., van de Velde, S.L. (2000) An agile planning and control framework for customer-order driven discrete parts manufacturing environments, International Journal of Agile Management Systems, pp. $16-23$. 
[13] Womack, J.P., Jones, D.T., Roos, D (1990) The machine that changed the world, New York : Rawson Associates, cop. 1990 